\title{
Severe Mosquito Bite Allergy
}

National Cancer Institute

\section{Source}

National Cancer Institute. Severe Mosquito Bite Allergy. NCI Thesaurus. Code C150489.

An EBV-positive NK-cell lymphoproliferative disorder characterized by high fever and intense local skin symptoms, including erythema, bullae, ulcers, skin necrosis, and deep scarring following mosquito bites. Patients have NK-cell lymphocytosis in the peripheral blood and an increased risk of developing hemophagocytic syndrome and progressing into overt NK/T-cell lymphoma or aggressive NK-cell leukemia in the longstanding clinical course. (WHO 2017) 